\title{
Changed cellular functions and aberrantly expressed miRNAs and circRNAs in bone marrow stem cells in osteonecrosis of the femoral head
}

\author{
SHUAI XIANG, ZENG LI and XISHENG WENG \\ Department of Orthopaedic Surgery, Peking Union Medical College Hospital, \\ Peking Union Medical College, Chinese Academy of Medical Science, Beijing 100730, P.R. China \\ Received January 14, 2019; Accepted May 15, 2019
}

DOI: $10.3892 /$ ijmm.2020.4455

\begin{abstract}
The present study aimed to detect the correlations between altered cellular functions in bone marrow stem cells (BMSCs) and osteonecrosis of the femoral head (ONFH). By profiling the aberrant expression of miRNAs and circRNAs in BMSCs isolated from ONFH patients, the present study aimed to further explore the potential regulatory mechanisms of action of circRNAs in ONFH using integrated bioinformatics analysis. BMSCs were isolated from seven ONFH patients and seven controls. Cellular functions, including proliferation, apoptosis and differentiation, were compared. miRNA and circRNA sequencing were conducted using RNA samples of three ONFH patients and three controls to identify differentially expressed circRNAs and miRNAs. The expression of hsa_circ_0000219, hsa_circ_0004588 and hsa_circ_0005936 were validated by qPCR. Target miRNAs were also predicted and validated by qPCR and circRNA-miRNA co-expression networks were constructed. BMSCs of ONFH patients displayed decreased proliferation and increased apoptosis during in vitro culturing. In addition, reduced osteogenesis and enhanced adipogenesis were found in the ONFH group. A total of 129 miRNAs and 231 circRNAs were detected to be differentially expressed. The expression levels of hsa_circ_0000219, hsa_circ_0004588 and hsa_circ_0005936 were significantly decreased in BMSCs of ONFH patients. A number of target miRNAs related to cell proliferation, apoptosis and differentiation were predicted for hsa_circ_0000219 and hsa_circ_0005936. The expression levels of miR-144-3p and miR-1270 were found to be elevated in ONFH patients, which was consistent
\end{abstract}

Correspondence to: Professor Xisheng Weng, Department of Orthopaedic Surgery, Peking Union Medical College Hospital, Peking Union Medical College, Chinese Academy of Medical Science, 1 Shuaifuyuan Wangfujing, Dongcheng, Beijing 100730, P.R. China E-mail: xishengweng@163.com

Key words: osteonecrosis of the femoral head, bone marrow stem cell, microRNA, circular RNA with miRNA sequencing data and competitive endogenous RNA hypothesis. Time-dependent expression patterns of hsa_circ_0000219, hsa_circ_0004588, hsa_circ_0005936, miR-144-3p and miR-1270 were also validated during osteogenic and adipogenic differentiation in BMSCs. The results of the present study substantiated the involvement of BMSCs in ONFH development. hsa_circ_0000219 and hsa_circ_0005936 may regulate the progression of ONFH by mediating the proliferation and differentiation of BMSCs by sponging miRNAs.

\section{Introduction}

Osteonecrosis of the femoral head (ONFH) leads to inestimable labor loss to the whole society with its high rate of disability (1). Typically, the disease becomes progressive and leads to severe pain and eventually, total hip arthroplasty is necessary for curing end-stage ONFH (2). However, the mechanism underlying the incidence and progression of ONFH remains largely unknown, limiting the advances in diagnosis and treatment for early-stage ONFH. Therefore, the underlying mechanisms of ONFH are valuable research topics that could guide the prevention, diagnosis and treatment of ONFH. In recent years, ONFH has been considered to be associated with the dysfunction of bone marrow stem cells (BMSCs). Previous studies have demonstrated well that decreased proliferation capacity, enhanced apoptosis, impaired osteogenesis and enhanced adipogenesis were found in BMSCs of ONFH patients $(3,4)$. In addition, aberrant gene expression was also found in BMSCs of ONFH patients and thus, is considered as the major cause of the dysfunction of BMSCs, particularly in the imbalance between osteogenesis and adipogenesis (5).

Increasing attention has been paid to detecting novel factors regulating gene expression in $\mathrm{ONFH}$ patients. Extensive efforts have been made on studying the function of non-coding RNAs (ncRNAs), especially microRNAs (miRNAs/miRs) (6). miR-199b-5p has been proved to promote osteogenesis of BMSCs by suppressing glycogen synthase kinase-3 $\beta / \beta$-catenin signaling pathway (7). miR-23a has been reported to inhibit osteogenic differentiation of BMSCs by targeting low-density lipoprotein receptor-related protein (8). However, it is inadequate to elucidate the pathogenesis of 
ONFH only by miRNAs. Previous studies have revealed that miRNAs could be dynamically regulated by other ncRNAs, such as circular RNAs (circRNAs) through a competing endogenous RNA (ceRNA) mechanism (9-11). Circular RNAs (circRNAs) are a large group of non-coding RNAs produced by non-canonical splicing of pre-mRNAs. Due to their continuous closed loop structures, circRNAs are resistant to RNA exonucleases or RNase R, leading to its stable and abundant presence in the cytoplasm, body fluids and blood (12). Nowadays, the advanced sequencing technology and bioinformatics analysis have facilitated the identification and classification of circRNAs. An increasing number of studies have investigated the regulatory function of circRNAs in diverse orthopedic diseases, including osteoarthritis (OA), rheumatoid arthritis (RA) and osteoporosis (13-15). However, research on the altered expression of circRNAs in BMSCs of ONFH patients is still lacking.

Based on previous studies, it was speculated that circRNAs were implicated in the pathogenesis of ONFH by regulating the functions of BMSCs, especially osteogenic and adipogenic differentiation $(16,17)$. In the present study, BMSCs were isolated from seven patients with steroid-induced ONFH and seven healthy controls and then the alteration in biological behavior of BMSCs in steroid-induced ONFH patients was investigated. In $\mathrm{ONFH}$ patients, a significantly reduced proliferation and osteogenesis of BMSCs was observed, along with enhanced apoptosis and adipogenesis. The expression of miRNA and circRNA in BMSCs was further screened using next-generation sequencing. The expression of three circRNAs and two miRNAs was validated by reverse transcription-quantitative (RT-q)PCR. Then, integrated bioinformatics methods were used to analyze the function of differentially expressed circRNAs and miRNAs. A circRNA-miRNA co-expression network was further constructed to detect the potential interaction between differentially expressed circRNAs and miRNAs. To the best of our knowledge, this study is the first to report the aberrant expression and function of circRNA in ONFH patients. These findings offer new insight into understanding the pathogenesis, facilitating early diagnosis and guiding treatment of this disease.

\section{Materials and methods}

Isolation, labeling and RNA extraction of BMSCs. Bone marrow tissue was acquired from seven female patients (age range, 26-54) with steroid-induced ONFH and seven female controls (age range, 26-75) who underwent hip arthroplasty in the Department of Orthopedics at Peking Union Medical College Hospital (Beijing, China) from June 2016 to December 2016. The inclusion criteria of the patient group included female ONFH patients with corticosteroids usage due to systemic lupus erythematosus (SLE). Patients with ONFH due to trauma, alcohol or corticosteroids use for treating other autoimmune diseases were excluded. Male patients were also excluded. Isolation and culturing of BMSCs was done using a method previously described (18). The growth medium used was a mixture of $440 \mathrm{ml}$ Dulbecco's modified Eagle medium (DMEM), $50 \mathrm{ml}$ fetal bovine serum (FBS; both Gibco; Thermo Fisher Scientific, Inc.), $5 \mathrm{ml}$ glutamine and $5 \mathrm{ml}$ penicillin-streptomycin solution. According to the manufacturer's protocol, total RNA of BMSCs was extracted using TRIzol agent (Invitrogen; Thermo Fisher Scientific, Inc.). Detailed information of the donors is listed in Table SI. Notably, the same RNA samples were simultaneously used for profiling and validating the long non-coding RNA and mRNA expression (not published yet). Surface markers including cluster of differentiation (CD)29, CD45 and CD90 were identified using mouse anti-human Alexa Fluor 647-CD29, CD45-fluorescein isothiocyanate (FITC), and CD90-FITC antibodies and analyzed by an Accuri C6 flow cytometer system.

This study was approved by the Ethics Committee at Peking Union Medical College Hospital and written consent was obtained from the all 14 tissue donors included in this study.

Proliferation and apoptosis assay. To investigate the proliferation ability, cells were seeded and cultured at a density of 5,000 cells in 96-well plates. Then, Cell Counting Kit-8 (CCK-8; Dojindo Molecular Technologies, Inc.) assay was performed after 24, 48 and $72 \mathrm{~h}$ of culturing. The Cell-Light ${ }^{\mathrm{TM}}$ EdU cell proliferation detection (EdU) assay (Guangzhou Ribobio Co., Ltd.) was performed $12 \mathrm{~h}$ after culturing, according to the manufacturer's protocol. Briefly, the cells were labeled with EdU. Subsequently, $100 \mu \mathrm{l}$ Apollo reaction cocktail and $100 \mu \mathrm{l}$ Hoechst 33342 solution were used in turn to stain the cells for $30 \mathrm{~min}$ on ice. Then the cells were visualized under a fluorescence microscope at a magnification of $\mathrm{x} 100$.

Cell apoptosis was analyzed by Annexin V-PI double staining after culturing the cells for $48 \mathrm{~h}$, according to the manufacturer's protocol. The cytographs were analyzed using FlowJo V10 software (FlowJo LLC). For each sample, the apoptosis assay was performed in triplicate.

Osteogenic and adipogenic differentiation in vitro. BMSCs were seeded in growth medium. Osteogenic medium (a mix of $175 \mathrm{ml}$ basal medium, $20 \mathrm{ml}$ FBS, $2 \mathrm{ml} \mathrm{b}$-glycerophosphate, $400 \mu \mathrm{l} \mathrm{L}$-ascorbic acid, $2 \mathrm{ml}$ glutamine, $2 \mathrm{ml}$ penicillin-streptomycin solution and $20 \mu \mathrm{l}$ dexamethasone) and adipogenic medium (A solution: A mix of $175 \mathrm{ml}$ basal medium, $20 \mathrm{ml}$ FBS, $2 \mathrm{ml}$ glutamine, $2 \mathrm{ml}$ penicillin-streptomycin solution, $400 \mu \mathrm{l}$ insulin, $200 \mu \mathrm{l}$ IBMX, $200 \mu \mathrm{l}$ rosiglitazone, and $200 \mu \mathrm{l}$ dexamethasone; B solution: A mix of $175 \mathrm{ml}$ basal medium, $20 \mathrm{ml} \mathrm{FBS}, 2 \mathrm{ml}$ glutamine, $2 \mathrm{ml}$ penicillin-streptomycin solution and $400 \mu \mathrm{l}$ insulin) were obtained from Cyagen Biosciences, Inc. For osteogenic differentiation, the growth medium was replaced with osteogenic differentiation medium when the cells reached $70-80 \%$ confluence. For adipogenic differentiation, growth medium was replaced with the adipogenic differentiation medium when cells reached $90-100 \%$ confluence. Subsequent procedures were done according to the protocol of Cyagen Biosciences, Inc.

Alizarin red staining and oil red $O$ staining. Alizarin red staining was conducted on day 12 after the osteogenic induction. In brief, cells were washed with PBS, fixed in $4 \%$ neutral buffered formalin for $1 \mathrm{~h}$ at room temperature, washed with PBS again and stained using alizarin red solution at $37^{\circ} \mathrm{C}$ for $30 \mathrm{~min}$.

Oil red $\mathrm{O}$ staining was performed on day 20 after adipogenic induction. The stock solution of Oil red $\mathrm{O}$ was diluted in deionized water and filtered prior to use. BMSCs were first washed with PBS, then fixed in $4 \%$ neutral buffered formalin 

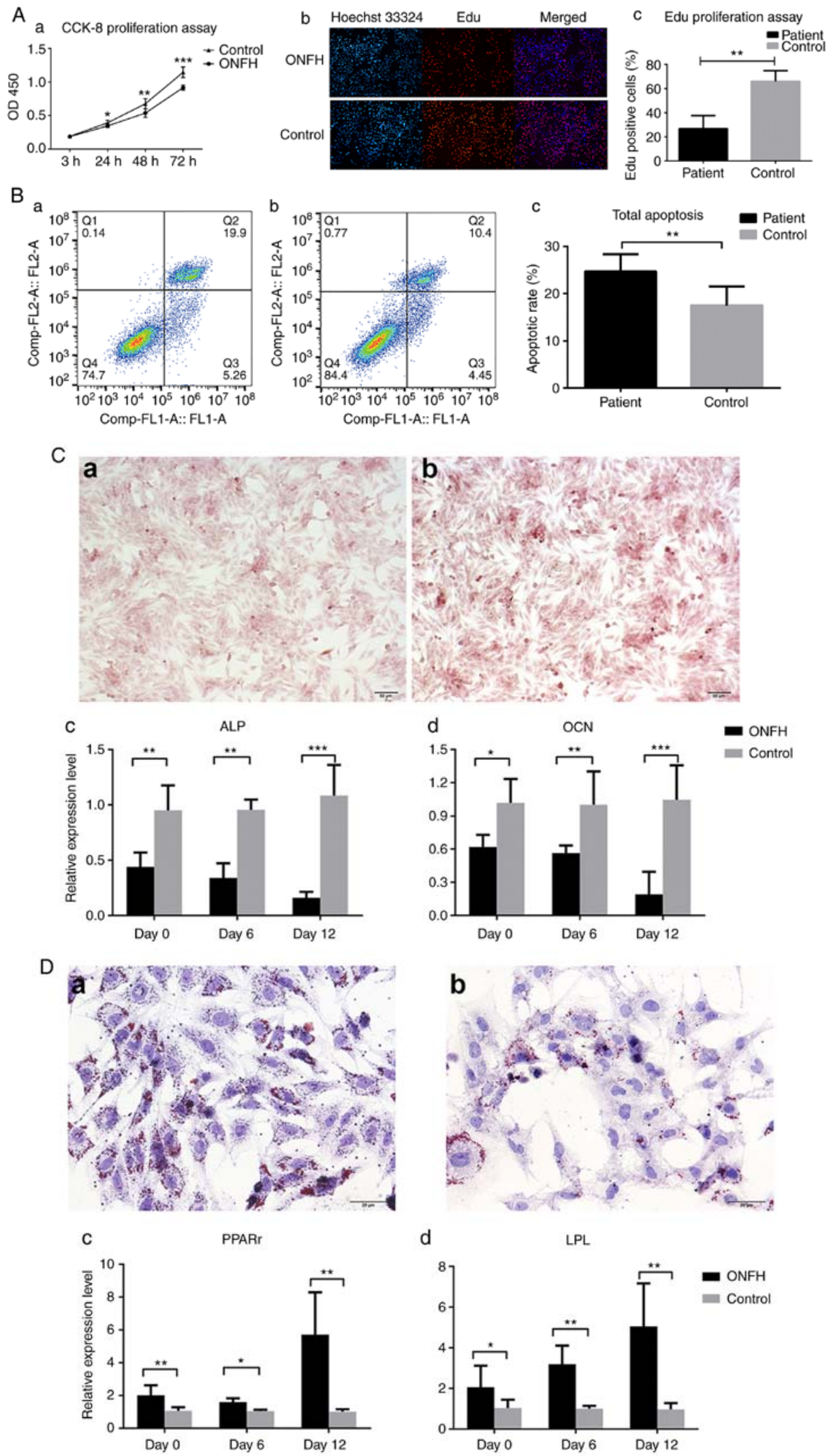

d

LPL

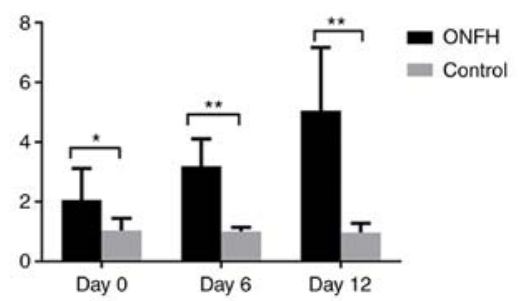

Figure 1. Altered cellular behavior in BMSCs in the ONFH group. (A) Decreased proliferation activity of BMSCs in ONFH patients was observed compared with the control group. (a) Decreased optical density at $450 \mathrm{~nm}$ was found in BMSCs in ONFH patients during in vitro expansion. (b) Representative image of EdU assay of BMSCs in ONFH patients (upper row) and controls (lower row). (c) The percentages of proliferating cells were quantified by EdU assay. (B) Cytographs of Annexin V-PI apoptosis assay of BMSCs in (a) ONFH patients and (b) controls. (c) Statistical analysis of total apoptotic cells. (C) Fewer calcium nodules revealed by alizarin red staining in BMSCs in (a) ONFH patients and (b) controls at day 12 of osteogenic differentiation (magnification, x40). Decreased expression of (c) ALP and (d) OCN in ONFH patients before and during the induction of osteogenic differentiation. (D) Accumulation of lipid granules in BMSCs in (a) ONFH patients and (b) controls, revealed by Oil red O staining at day 20 of the induction of adipogenic differentiation (magnification, $x 100$ ). Increased expression of (c) LPL and (d) PPAR $\gamma$ in ONFH group before and during the induction of adipogenic differentiation. ${ }^{*} \mathrm{P}<0.05,{ }^{* * *} \mathrm{P}<0.01$ and ${ }^{* * *} \mathrm{P}<0.001$. BMSCs, bone marrow stem cells; ONFH, osteonecrosis of the femoral head; PI, propidium iodide; PPAR, peroxisome proliferator-activated receptor; ALP, alkaline phosphatase; LPL, lipoprotein lipase. 
for $1 \mathrm{~h}$ at room temperature, washed with deionized water and stained with Oil red $\mathrm{O}$ solution for $30 \mathrm{~min}$ at room temperature. Excess Oil red $\mathrm{O}$ stain was washed away with $60 \%$ isopropanol. The staining results were observed and recorded under a light microscope at a magnification of $\mathrm{x} 100$.

Sequencing and differential expression analysis of miRNA and circRNA. After evaluating the RNA integrity, concentration and purity, sequencing procedures were performed by Annoroad Gene Technology, Co., Ltd. Briefly, ribosomal RNA was first removed using Epicentre Ribo-Zero ${ }^{\mathrm{TM}}$ Gold kits (Epicentre; Illumina, Inc.) and generated sequencing libraries following manufacturer's protocols with varied index label using NEBNext ${ }^{\circledR}$ Ultra $^{\mathrm{TM}}$ Directional RNA Library Prep kit from Illumina, Inc. Then the libraries were sequenced on an Illumina Hiseq 4000 platform and $150 \mathrm{bp}$ paired-end reads were generated. Raw Data acquired was processed with Perl scripts to ensure the quality of data. CIRI 1.2 software was used to recognize circRNA (19). Spliced Reads per Billion Mapping was then used to quantify the expression of each circRNA (20). MiRDeep2 (21) was used to identify miRNA and Transcripts Per Million was used for estimation and normalization. Then, DEGseq v1.18.0 was used for differential expression analysis of miRNA and circRNA. The P-value was adjusted by the Benjamini and Hochberg's approach for controlling the false discovery rate. Genes with adj. $\mathrm{P} \leq 0.05$ and $\mid \log 2$ _foldchangel $\geq 1$ were identified as differentially expressed (DE) circRNA and DE miRNA.

$R T-q P C R$. This analysis was conducted prior to the induction of differentiation as well as on day 3,6 , and 12 of the induction. PrimeScript ${ }^{\mathrm{TM}}$ RT reagent kit with gDNA Eraser (Takara Bio, Inc.) was used for reverse transcription of RNA samples. The reaction was performed at $37^{\circ} \mathrm{C}$ for $15 \mathrm{~min}$ and then at $85^{\circ} \mathrm{C}$ for $5 \mathrm{sec}$. qPCR was performed using $\mathrm{SYBR}^{\circledR}$ Premix Ex Taq ${ }^{\mathrm{TM}}$ II (Tli RNaseH Plus), ROX plus (Takara Bio, Inc.) for the analysis of hsa_circ_0000219, hsa_circ_0004588, hsa_circ_0005936, alkaline phosphatase (ALP), osteocalcin $(\mathrm{OCN})$, peroxisome proliferator-activated receptor $\gamma(\operatorname{PPAR} \gamma)$ and lipoprotein lipase (LPL). The thermocycling conditions were as follows: Firstly, $95^{\circ} \mathrm{C}$ for $10 \mathrm{~min}$; then 40 cycles of $95^{\circ} \mathrm{C}$ for $15 \mathrm{sec}, 60^{\circ} \mathrm{C}$ for $45 \mathrm{sec}$ and $70^{\circ} \mathrm{C}$ for $30 \mathrm{sec}$. GAPDH mRNA was set as internal controls for circRNAs and mRNAs. U6 mRNA was set as internal controls for miRNAs. The relative expression of each DEG was calculated using the $2^{-\Delta \Delta \mathrm{Cq}}$ method (22). Reactions of each sample were run in triplicate. miRNAs primers were purchased from Guangzhou RiboBio, Co., Ltd., and gene primers are displayed in Table SII.

Functional analysis of DEGs. The Gene Ontology (GO; http://geneontology.org/) and Kyoto Encyclopedia of Genes and Genomes (KEGG; http://www.kegg.jp/) analysis of DE circRNAs and DE miRNAs was implemented by the hypergeometric test. GO and KEGG terms with adj. $\mathrm{P}<0.05$ was considered to indicate significant enrichment.

Prediction of miRNA target and circRNA-miRNA interaction. MiRanda 2010 Release (http://www.microrna. org/microrna/home.do), PITA v6 (http://genie.weizmann. ac.il/pubs/mir07/mir07_dyn_data.html) and TargetScan 7.0 (http://www.targetscan.org/) were used for predicting target genes of known or novel miRNAs. To identify target miRNAs of selected DE circRNAs, Arraystar's home-made software v1.0 (23), based on TargetScan and miRanda, was used. The predicted miRNAs were further compared to the list of DE miRNAs and then, a circRNA-miRNA co-expression network was constructed based on competitive endogenous RNA principle. Cytoscape 3.4.0 software (https://cytoscape. org) was used to visualize the circRNA-miRNA network.

Statistical analysis. All data in this study were presented as the mean \pm standard deviation and each experiment was performed in triplicate independently. Prism 7 software (GraphPad Software, Inc.) was employed to perform the statistical analysis. A two-tailed t-test was used to compare the difference between two groups and comparisons between multiple groups were done by Kruskal-Wallis test along with Dunnett's multiple comparisons test. $\mathrm{P}<0.05$ was considered to indicate a statistically significant difference.

\section{Results}

Phenotype and altered cellular behaviors of BMSCs in ONFH patients. The isolated BMSCs displayed a swirling arranged phenotype microscopically (Fig. S1). A total of three surface antigens, including CD29, CD45 and CD90 were further investigated by flow cytometry analysis (Fig. S1B-D). BMSCs from passage 3 were positive for CD29 and CD90 (89.5 and 99.9\%, respectively) and negative for CD45 $(0.16 \%)$.

The cellular behaviors between BMSCs of patient and control groups were then compared. A significant decrease in proliferation of BMSCs of ONFH patients by CCK-8 and EdU proliferation assays were identified $(\mathrm{P}<0.05$; Fig. 1A). It was also found that the total apoptotic rate of BMSCs of ONFH patients was elevated (Fig. 1B).

Alizarin red staining and oil red $\mathrm{O}$ staining was performed to compare the differentiation capacity of the cells. BMSCs from the ONFH group exhibited significantly decreased calcium mineralization and increased lipid accumulation in the cytoplasm ( $\mathrm{P}<0.05$; Fig. 1C-a, C-b, D-a and D-b). Consistent with the results of staining, the lower expression of osteogenic markers ALP and OCN as well as enhanced expression of adipogenic markers PPAR $\gamma$ and LPL was found during the process of osteogenic and adipogenic induction, respectively (Fig. 1C-a, C-b, D-a and D-b).

The miRNA and circRNA expression profile in ONFH patients. Overall, 897 miRNAs and 14,131 circRNAs were screened. miRNAs and circRNAs with $\mid \log 2$ _foldchangel $\geq 1$ and adj. $\mathrm{P} \leq 0.05$ were considered as differentially expressed. A total of 129 DE miRNAs were detected, of which 47 miRNAs were downregulated and 82 miRNAs were upregulated in BMSCs of ONFH patients. In addition, there were 231 DE circRNAs, including 141 upregulated circRNAs and 90 downregulated circRNAs. Among them, 215 circRNAs were transcribed from the exonic region and only 16 from the intronic region or the intergenic region. The expression profiles of miRNAs and circRNAs are displayed in Figs. 2 and 3. Top 10 up- and downregulated miRNAs and circRNAs are listed in Tables I and II. 
A

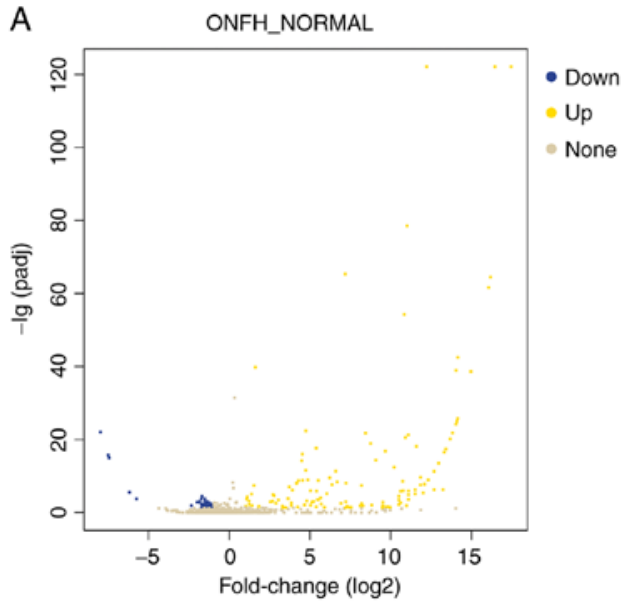

B

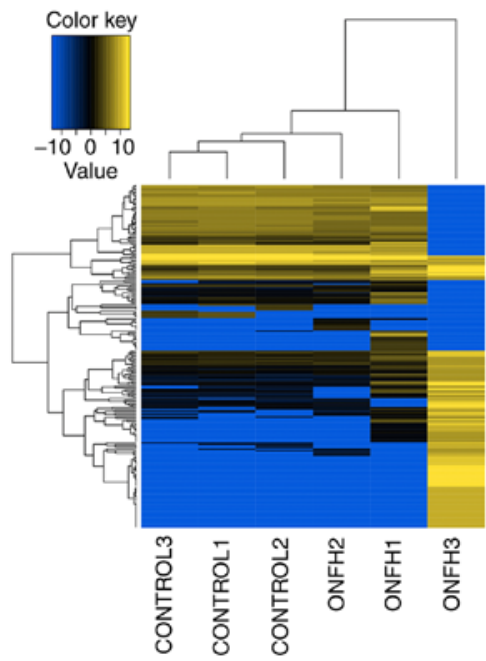

Figure 2. The expression profiles of miRNAs DE miRNAs between BMSCs of ONFH patients and controls. (A) Volcano plots displays 129 DE miRNAs with adj. $P \leq 0.05$ and $l \log 2 \_$ratiol $\geq 1$. Yellow points represent upregulated miRNAs and blue points represent downregulated ones. (B) In hierarchical clustering analysis, the color depth indicated the elevated (yellow) and decreased (blue) expression levels. BMSCs, bone marrow stem cells; ONFH, osteonecrosis of the femoral head; DE, differentially expressed; miRNA, microRNA.

A

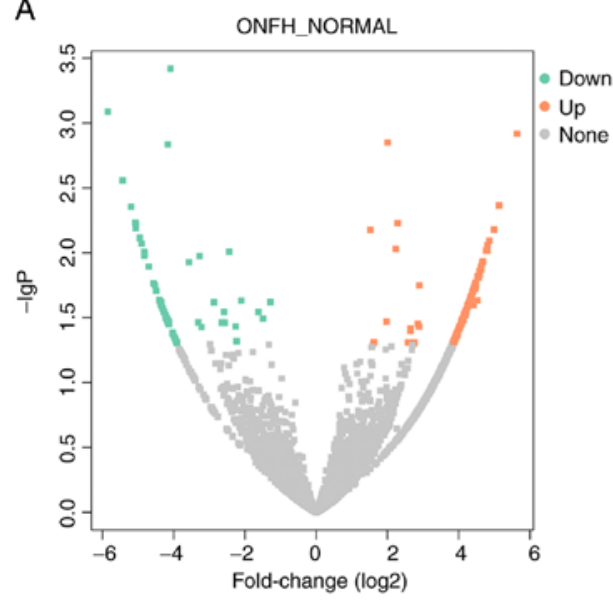

C

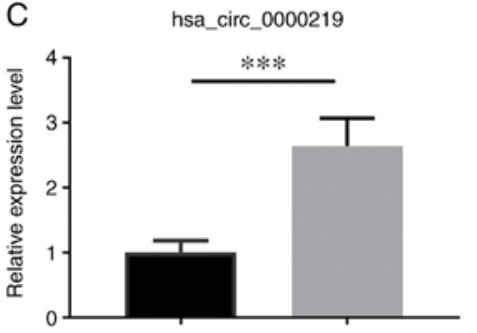

B

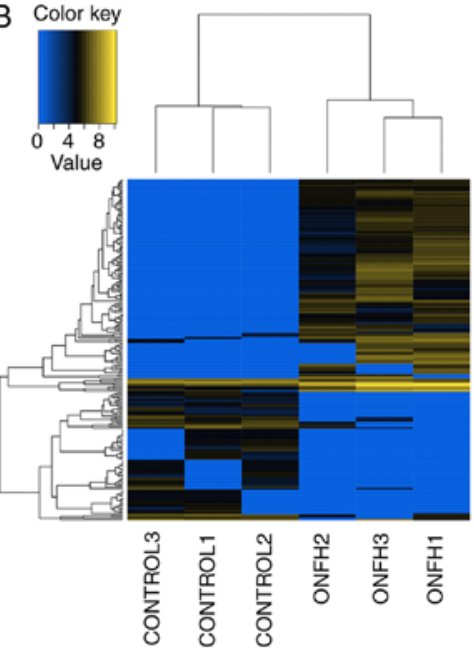

D

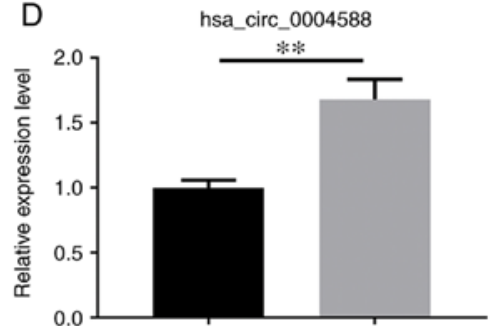

E

hsa_circ_0005936

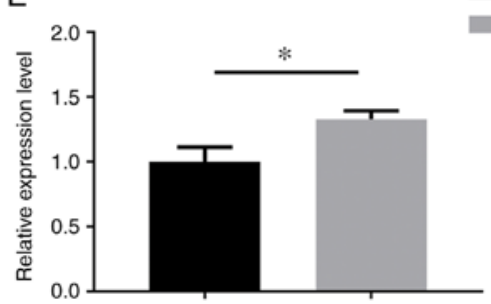

Figure 3. The expression profiles of DE circRNAs in BMSCs in ONFH patients. (A) Volcano plots displays 231 DE circRNAs with adj. P $\leq 0.05$ and llog2_ratiol $\geq 1$. Yellow points represent upregulated circRNAs and blue points represent downregulated ones. (B) In hierarchical clustering analysis, the color depth indicated the elevated (yellow) and decreased (blue) expression levels. Significantly decreased expression of circRNAs, (C) hsa_circ_0000219, (D) hsa_circ_0004588 and (E) hsa_circ_0005936 in BMSCs of ONFH patients ( $\mathrm{n}=7$ ) compared with in the control group $(\mathrm{n}=7)$. ${ }^{*} \mathrm{P}<0.05,{ }^{* * *} \mathrm{P}<0.01$ and ${ }^{* * * *} \mathrm{P}<0.001$. circ, circular; BMSCs, bone marrow stem cells; ONFH, osteonecrosis of the femoral head; DE, differentially expressed. 
Table I. Top 10 up- and downregulated miRNAs.

A, Upregulated miRNAs

\begin{tabular}{lcc}
\hline Gene & Log2Fold-change & adj. P-value \\
\hline hsa-miR-4451 & 14.043 & $1.16 \times 10^{-39}$ \\
hsa-miR-6757-5p & 13.25041 & $5.50 \times 10^{-07}$ \\
hsa-miR-7114-3p & 12.68606 & $5.64 \times 10^{-07}$ \\
hsa-miR-124-3p & 12.00992 & $3.98 \times 10^{-06}$ \\
hsa-miR-4787-5p & 11.59704 & $7.07 \times 10^{-19}$ \\
hsa-miR-1270 & 11.09448 & $4.84 \times 10^{-22}$ \\
hsa-miR-6750-5p & 10.97533 & 0.014683 \\
hsa-miR-6800-3p & 10.97533 & 0.014683 \\
hsa-miR-6089 & 10.91785 & $2.69 \times 10^{-21}$ \\
hsa-miR-9-5p & 10.83323 & $5.56 \times 10^{-55}$ \\
\hline
\end{tabular}

B, Downregulated miRNAs

\begin{tabular}{lll}
\hline hsa-miR-30a-5p & -1.53456 & 0.022986 \\
hsa-miR-582-5p & -1.60469 & 0.004317 \\
hsa-miR-496 & -1.67512 & 0.020004 \\
hsa-miR-199b-5p & -1.69292 & $3.71 \times 10^{-05}$ \\
hsa-miR-302d-3p & -1.7246 & 0.041531 \\
hsa-miR-31-3p & -1.73719 & 0.000222 \\
hsa-miR-628-5p & -1.74575 & 0.003749 \\
hsa-miR-154-5p & -1.84565 & 0.001145 \\
hsa-miR-30c-2-5p & -1.99482 & 0.001591 \\
hsa-miR-549a & -2.3367 & 0.014287 \\
\hline
\end{tabular}

miR/miRNA, microRNA.

Validation of the circRNA profile by RT-qPCR. A total of 3 downregulated circRNAs (hsa_circ_0000219, hsa_ circ_0004588 and hsa_circ_0005936) were selected for verification of the hypothesis that downregulated circRNAs could potentially become genetic tools in early treatment for steroid-induced ONFH by bone tissue engineering. The expression of the selected circRNAs was examined in BMSCs from seven patients with steroid-induced ONFH and seven controls using RT-qPCR. Consistent with the authors' sequencing data, the expression levels of hsa_circ_0000219, hsa_circ_0004588 and hsa_circ_0005936 were all significantly decreased in BMSCs from ONFH patients compared with the controls $(\mathrm{P}<0.05$; Fig. 3C-E), indicating a potential positive correlation between the expression of these circRNAs and proliferation and osteogenic capacity of BMSCs, as well as a negative correlation between circRNAs expression and apoptosis and adipogenic capacity of BMSCs.

GO and KEGG analysis of DE miRNAs and DE circRNAs. A total of 27,329 target genes were predicted by at least two pieces of software for the validation of DE miRNAs and the potential function of the DE miRNAs were was identified by GO analysis and KEGG analysis of the target genes. Collectively, 1,886 biological process (BP) terms, 182 cellular component (CC) terms and 237 molecular function (MF) terms were considered significant, along with 129 KEGG terms. Table SIII displays the 10 most significant BP, MF, and KEGG terms for the DE miRNAs. The function of DE circRNAs was represented by the functional analysis of their parental genes. Based on the results of the present study, a total of $602 \mathrm{BP}$ terms, $44 \mathrm{CC}$ terms, $140 \mathrm{MF}$ terms and $11 \mathrm{KEGG}$ pathways were identified as significant. Detailed information is shown in Table SIV.

Target miRNAs prediction and verification for validated circRNAs. Based on TargetScan and miRanda, the target miRNAs of the three validated downregulated circRNAs were predicted and further compared with the results of miRNA sequencing. A number of upregulated DE miRNAs were predicted as targets for hsa_circ_0000219 and hsa circ_0005936 (Fig. 4A and B). However, no circRNA-miRNA interactions were predicted for hsa_circ_0004588. Therefore, miR-144-3p was selected, a predicted target miRNA for hsa_circ_0000219 reported to inhibit the proliferation and osteogenic differentiation of BMSCs, and miR-1270, a common target for both hsa_circ_0000219 and hsa_circ_0005936 for further verification (Fig. 4C and D). Consistent with the sequencing data of the present study, these two miRNAs were both significantly upregulated in BMSCs of ONFH patients, indicating a potential ceRNA mechanism-mediated regulation of progression of steroid-induced ONFH ( $\mathrm{P}<0.001$; Fig. 4E).

Time-dependent expression patterns of DE circRNAs and miRNAs during differentiation of BMSCs. Considering that the imbalance of osteogenic and adipogenic differentiation of BMSCs outweighs their proliferation and apoptosis in the progression of ONFH, whether the selected circRNAs and miRNAs could potentially regulate differentiation of BMSCs was examined. BMSCs were induced with osteogenic medium and adipogenic medium for 12 days and the expression of circRNAs and miRNAs was detected on day 3, 6, and 12 of induction with RT-qPCR. The expression of hsa_circ_0000219 and hsa_circ_0005936 consistently decreased during the osteogenic induction and reached significance on day 12 $(\mathrm{P}<0.05$; Fig. 5A and B). During the adipogenic differentiation, significantly elevated expression of hsa_circ_0000219 and hsa_circ_0005936 was also found on day 12 ( $\mathrm{P}<0.05$; Fig. 5A and B). However, the relationship of hsa_circ_0004588 and differentiation of BMSCs was not detected. The expression of miR-144-3p increased during osteogenic differentiation and decreased during adipogenic differentiation, displaying a negative relationship with hsa_circ_0000219 and hsa_ circ_0005936 (Fig. 5C).

\section{Discussion}

Although the pathogenesis of $\mathrm{ONFH}$ remains elusive, several studies have demonstrated the association of aberrant behavior of BMSCs with the development of ONFH, as well as their promising roles in treatment for $\mathrm{ONFH}$ via bone tissue engineering $(3,24,25)$. In the present study, BMSCs were isolated from patients with ONFH and controls and the surface markers were checked. CD29 is the marker of mesenchymal cells and CD90 is the marker of stem 
Table II. Top 10 up- and downregulated circRNAs and their source genes.

A, Downregulated circRNAs

\begin{tabular}{|c|c|c|c|}
\hline Gene & Log2Fold-change & Adj. P-value & Source_Gene \\
\hline hsa_circ_0013180 & -5.633964 & 0.001209 & Null \\
\hline hsa_circ_0011021 & -5.133924 & 0.004323 & ENSG00000096060 \\
\hline hsa_circ_0000761 & -4.988706 & 0.006605 & ENSG00000182985 \\
\hline hsa_circ_0010245 & -4.857244 & 0.008042 & ENSG00000112972 \\
\hline hsa_circ_0006469 & -4.812017 & 0.00875 & ENSG00000087586 \\
\hline hsa_circ_0003004 & -4.809939 & 0.009577 & ENSG00000092439 \\
\hline hsa_circ_0000219 & -4.77287 & 0.009605 & ENSG00000015171 \\
\hline hsa_circ_0000596 & -4.676935 & 0.011608 & ENSG00000107771 \\
\hline hsa_circ_0005936 & -4.674644 & 0.011719 & ENSG00000117419 \\
\hline hsa_circ_0012564 & -4.650421 & 0.012237 & ENSG00000066697 \\
\hline
\end{tabular}

B, Upregulated circRNAs

\begin{tabular}{|c|c|c|c|}
\hline hsa_circ_0010461 & 4.82842 & 0.008508 & ENSG00000164176 \\
\hline hsa_circ_0001854 & 4.90173 & 0.007764 & ENSG00000058272 \\
\hline hsa_circ_0002614 & 4.94959 & 0.007629 & ENSG00000126773 \\
\hline hsa_circ_0003535 & 4.95424 & 0.006065 & ENSG00000174939 \\
\hline hsa_circ_0004764 & 5.05521 & 0.006463 & ENSG00000237440 \\
\hline hsa_circ_0011258 & 5.06074 & 0.005875 & ENSG00000118412 \\
\hline hsa_circ_0005623 & 5.07195 & 0.005843 & ENSG00000116991 \\
\hline hsa_circ_0011690 & 5.07232 & 0.004407 & ENSG00000106571 \\
\hline hsa_circ_0003795 & 5.19475 & 0.002763 & ENSG00000065559 \\
\hline hsa_circ_0002691 & 5.43192 & 0.000816 & ENSG00000119707 \\
\hline hsa_circ_0010193 & 5.84601 & 0.008508 & ENSG00000164190 \\
\hline
\end{tabular}

Circ, circular.

cells. The absence of CD45 excluded pan-hematopoietic cells (26). The combination of the three markers in this study confirmed the BMSCs were successfully isolated. Despite the limited number of cases, it was confirmed that the proliferation capacity of BMSCs decreased in ONFH patients, along with enhanced apoptosis. Reduced osteogenic and increased adipogenic capacity of BMSCs in ONFH patients was also verified. The results of the present study substantiated previous studies regarding behaviors of BMSCs in steroid-induced ONFH $(3,4)$.

Aberrant gene expression has been identified in BMSCs of ONFH patients and is considered to regulate the altered cellular functions. Investigation of the regulation of gene expression was emphasized and a large number of ncRNAs, especially miRNAs were detected to be aberrantly expressed in ONFH patients. These miRNAs were reportedly implicated in the progression of ONFH mainly through the regulation of differentiation of BMSCs (27). Due to the advancement in sequencing technology and bioinformatics analysis, circRNAs, which have long been considered as a by-product produced during RNA splicing, have been emerged as new hotspot in ONFH (28). Their stability makes them suitable to be biomarkers and tools for gene therapy (12).
circRNAs could bind miRNAs and function through a ceRNA mechanism (29). Unfortunately, only a few studies have focused on the roles of circRNAs in ONFH $(17,30)$. In the present study, the expression pattern of circRNAs and miRNAs were profiled simultaneously by high-throughput RNA-sequencing. A total of 231 DE circRNAs and 129 DE miRNAs were detected. Considering that downregulated circRNAs could become potential tools used to rescue the impaired proliferation and osteogenic capacity of BMSCs, while promoting bone repair in necrotic area, three downregulated circRNAs, hsa_circ_0000219, hsa_circ_0004588, and hsa_circ_0005936, were randomly selected to validate their expression levels by qPCR. Compared with BMSCs of the control group, the expression of hsa_circ_0000219, hsa_circ_0004588 and hsa_circ_0005936 was decreased in BMSCs of the ONFH group, indicating the involvement of these downregulated circRNAs in ONFH. Furthermore, target miRNAs were predicted for validated circRNAs and a circRNA-miRNA network was constructed based on the ceRNA principle (31). Although no circRNA-miRNA interaction was predicted for hsa_circ_0004588, a total of 17 upregulated miRNAs were found to be targets for hsa_circ_0000219 and hsa_circ_0005936. 
A

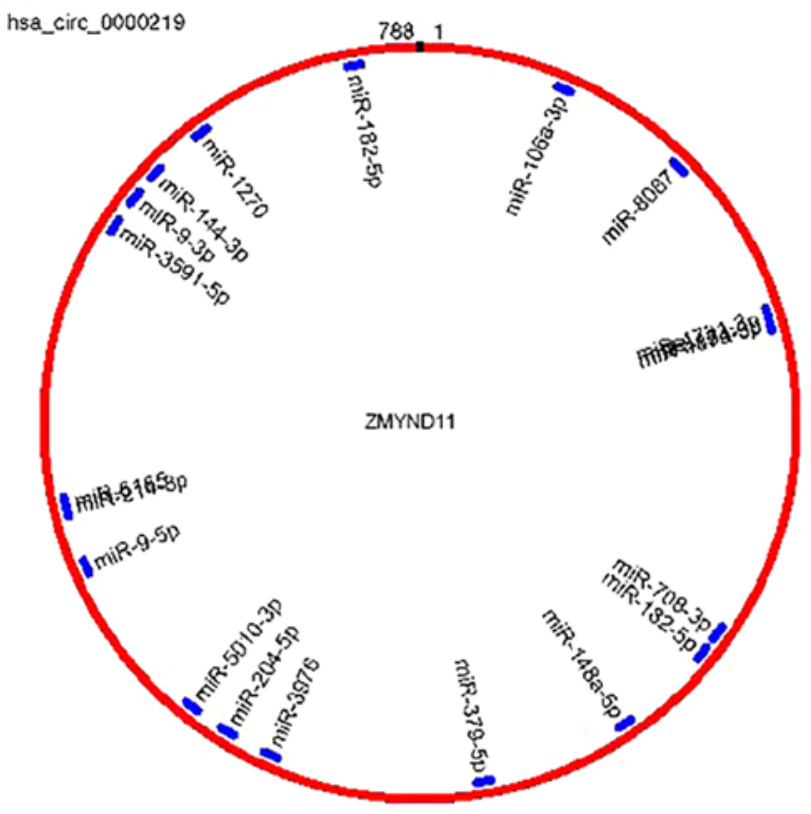

C
B
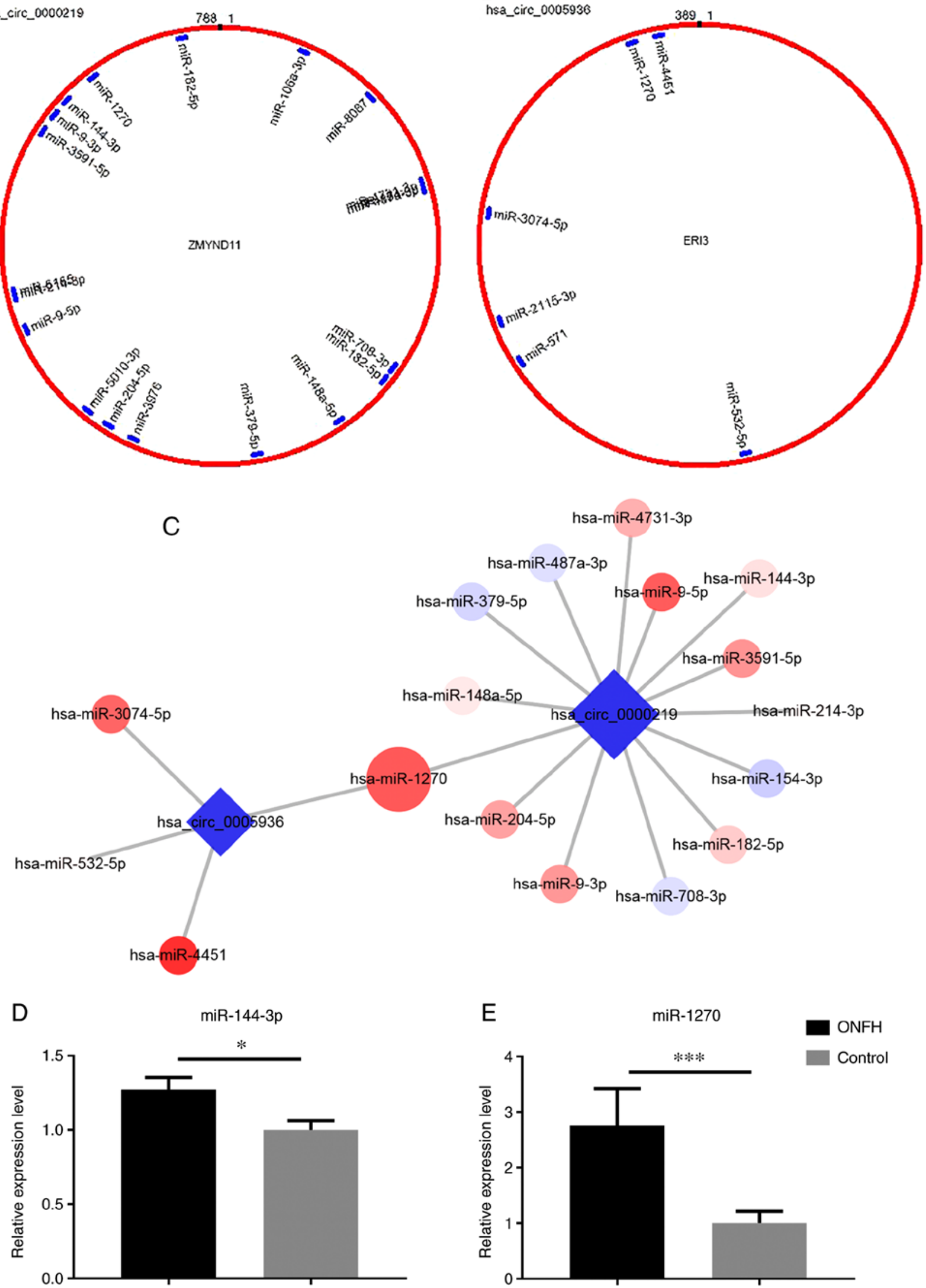

hsa-miR-4731-3p

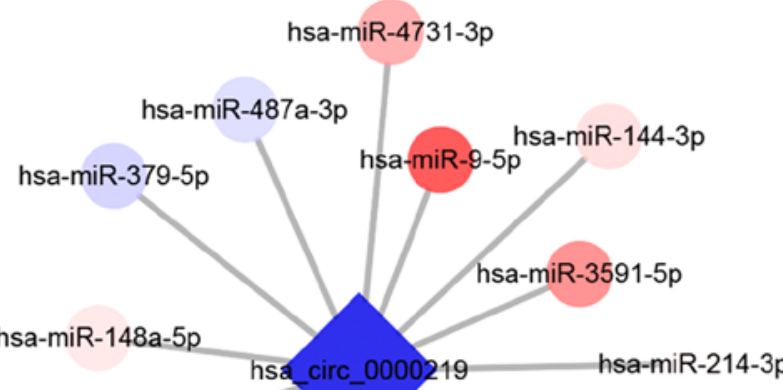
hsa-miR-204-5p
hsa-miR-9-3p hsa-miR-708-3p

hsa-miR-182-5p

$E$

$\operatorname{miR}-1270$

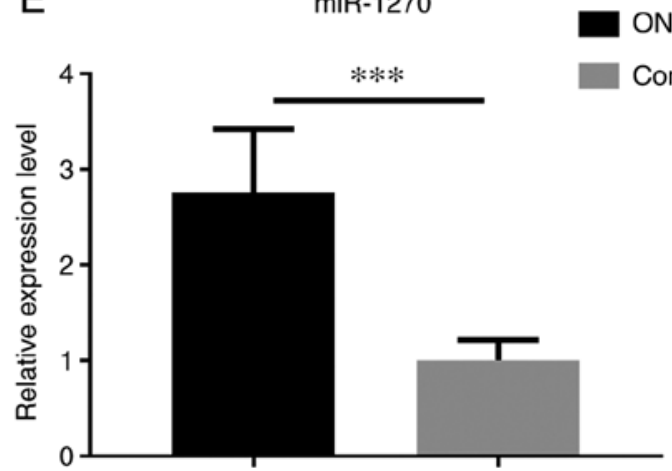

Figure 4. Target miRNAs prediction and validation for hsa_circ_0000219 and hsa_circ_0005936. (A) Structure and predicted target miRNAs of hsa_circ_0000219. (B) Structure and predicted target miRNAs of hsa_circ_0005936. (C) CircRNA-miRNA co-expression network of hsa_circ_0000219, hsa_circ_0005936 and their target miRNAs. Diamond nodes represent circRNAs and round nodes represent miRNAs. The intensity of red (upregulated) or blue (downregulated) indicates the fold-change of circRNAs and miRNAs. Two predicted target miRNAs, (D) miR-144-3p and (E) miR-1270 significantly elevated in BMSCs in ONFH group $(n=7)$ compared with the control group $(n=7)$. ${ }^{*} \mathrm{P}<0.05$ and ${ }^{* * *} \mathrm{P}<0.001$. miR/miRNA, microRNA; circ, circular; ONFH, osteonecrosis of the femoral head. 
A

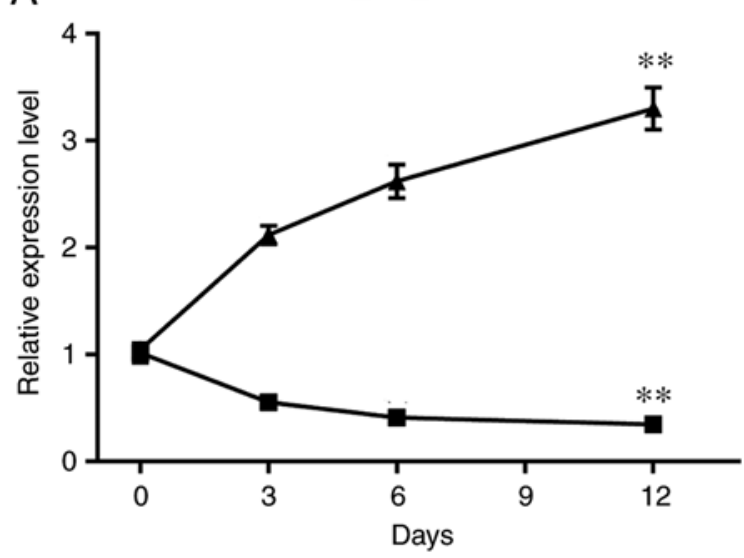

B hsa_circ_0005936

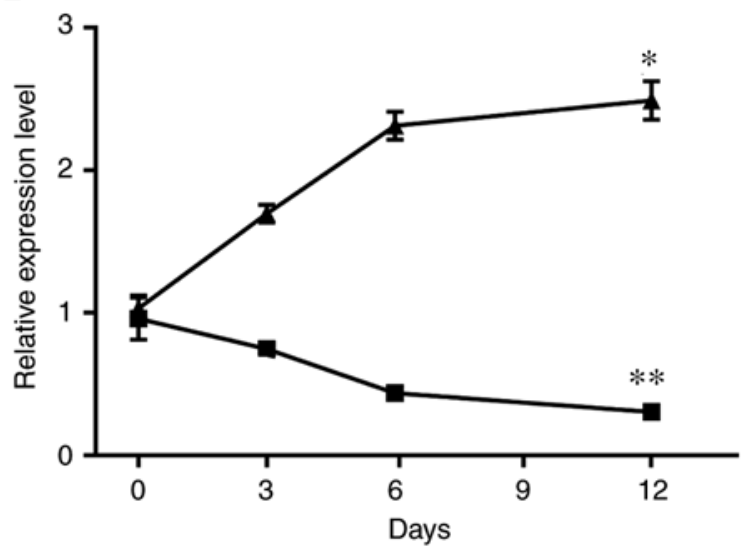

C

miR-144-3p

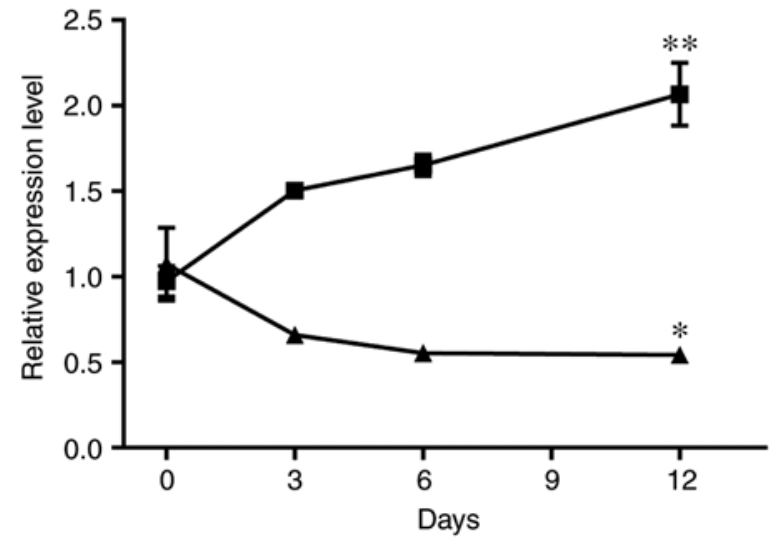

Osteogenic differentiation

Adipogenic differentiation

Figure 5. The time-dependent expression pattern of hsa_circ_0000219, hsa_circ_0005936 and miR-144-3p. (A) The expression of hsa_circ_0000219 was significantly elevated during osteogenic differentiation and decreased during adipogenic differentiation. (B) The expression of hsa_circ_0005936 was significantly elevated during osteogenic differentiation and decreased during adipogenic differentiation. (C) The expression of miR-144-3p was significantly elevated during adipogenic differentiation and decreased during osteogenic differentiation. ${ }^{*} \mathrm{P}<0.05$ and ${ }^{* *} \mathrm{P}<0.01$. miR, microRNA.

Since the functions of the selected circRNAs in ONFH have not been reported yet, the authors speculated their functions through the source genes and target miRNAs. Hsa_circ_0000219 is located at chr10: 179994-221356 and its parental gene is zinc finger MYND-type containing 11 , whose mutation was found to be associated with acute poorly differentiated myeloid leukemia (32), indicating its potential involvement in cell differentiation. However, the source genes of hsa_circ_0004588 and hsa_circ_0005936 are phosphatidylinositol glycan anchor biosynthesis class $\mathrm{N}$ and exoribonuclease family member 3 , respectively, whose functions have been rarely reported previously. Although the functions of most target miRNAs of hsa_circ_0000219 and hsa_circ_0005936 have been reported in cancer instead of BMSCs, the present study speculated their functions in BMSCs using previous literature. Only one study on miR-9a-5p reported that the target gene of miR-9a-5p is Sirt1 (33). The expression of Sirt1 in BMSCs promotes proliferation, inhibits apoptosis and pushes the direction of differentiation towards the osteogenic lineage $(34,35)$. miR-379-5p directly targets 3'-UTR of focal adhesion kinase (FAK) and FAK loss has adverse effects on proliferation and osteogenic differentiation of preosteoblasts by repressing Wnt/ $/$-catenin signaling $(36,37)$. miR-214-3p overexpression suppresses cell proliferation in gastric cancer by targeting runt-related transcription factor (RUNX3). In mesen- chymal stem cell (MSCs), RUNX3 plays an important role in mediating the bone morphogenic protein (BMP)9-induced osteogenic differentiation $(38,39)$. miR-144-3p inhibits the proliferation and osteogenic differentiation in MSC lines C3H10T1/2, by directly targeting mothers against decapentaplegic homolog 4 (40). Forkhead box $\mathrm{C} 1$ has been reported to be a target gene of miR-204-5p and is a crucial regulator of early osteogenic differentiation induced by BMP4 $(41,42)$. The target miRNA of hsa_circ_0005936, miR-3074-5p, promotes cell apoptosis (43). Taken together, all the target miRNAs were upregulated and impaired the proliferation and osteogenic capacity while promoting cell apoptosis of BMSCs during the progression of ONFH. Therefore, miR-144-3p was selected, whose function has been well documented and miR-1270, a target miRNA for both hsa_circ_0000219 and hsa_circ_0005936, to preliminarily validate their expression by qPCR. The results showed that the expression levels of these two miRNAs were elevated in BMSCs of ONFH patients. The results of the present study indicated that the downregulation of hsa_circ_0000219 and hsa_circ_0005936 may contribute to ONFH progression by mainly affecting the differentiation of BMSCs, through the hsa_circ_0000219-miR-144-3p or hsa_circ_0005936-miR-1270 axis. However, more studies are needed to elucidate the exact functions of the circRNAs and to evaluate their potential in early interference in ONFH. 
However, this study has certain limitations. First, the conclusion may be compromised due to the limited number of patients and control donors, and more samples are being collected to further substantiate these conclusions. Second, whether there are differentially expressed circRNAs between old trauma fracture or DDH and trauma-related ONFH has not been proved. Thus, the differentially expressed genes might result from loss of blood supply instead of ONFH itself. Basically, loss of blood supply has been proved to be one of the most important pathological changes in ONFH of all causes. Thus, it was speculated that the DE circRNAs or miRNAs associated with blood loss could also be correlated with ONFH. Third, the DE circRNAs and miRNAs might also result from the autoimmune diseases such as SLE instead of $\mathrm{ONFH}$, which has been demonstrated in previous studies (44-46). It is true that SLE itself could lead to DE miRNAs and circRNAs. However, whether SLE leads to functional alteration in BMSCs is still unclear. In the current study, bone marrow tissue was collected from the proximal region of the femur during hip replacement surgery, thus, BMSCs of SLE patients without ONFH were not obtained, which made this problem hard to answer. However, the time-dependent expression of two circRNAs and a miRNA was detected in BMSCs to demonstrate that they are associated with differentiation. Although it still cannot be known whether the DE circRNAs and miRNAs related to SLE or steroid treatment, it can be concluded that they are related to aberrant differentiation of BMSCs, which has been proved in ONFH.

In the present study, BMSCs were isolated from seven ONFH patients and seven controls, and profiled the expression levels of circRNA in BMSCs of ONFH patients for the first time. Then, the sequencing data of circRNA and miRNA were comprehensively analyzed. Several signaling pathways regulating multiple cellular functions were screened during functional analysis. circRNA-miRNA co-expression networks were also constructed. A total of three circRNAs and their predicted miRNAs were validated by qPCR in BMSCs. The results of the present study could improve understanding of the regulatory roles of circRNA-miRNA interaction in ONFH development and provide new insights for early treatment of ONFH using BMSCs and circRNAs as genetic tools.

\section{Acknowledgements}

Not applicable.

\section{Funding}

The present study is supported by the National Natural Science Foundation of China (grant no. 81572143 and 81630064).

\section{Availability of data and materials}

The datasets used and/or analyzed during the current study are available from the corresponding author on reasonable request.

\section{Authors' contributions}

SX performed the experiments and analyzed the results. ZL was a major contributor in writing the manuscript, and also helped in to perform several experiments and analyzing the results. XW designed the research and was a major contributor in recruiting the donors. All authors read and approved the final manuscript.

\section{Ethics approval and consent to participate}

This study was approved by the Ethics Committee at Peking Union Medical College Hospital and written consent was obtained from the all 14 tissue donors included in this study.

\section{Patient consent for publication}

All donors included in the current study provided informed consent for publication at the point of recruitment.

\section{Competing interests}

The authors have declared that no conflict of interest exists.

\section{References}

1. Cui L, Zhuang Q, Lin J, Jin J, Zhang K, Cao L, Lin J, Yan S, Guo W, He W, et al: Multicentric epidemiologic study on six thousand three hundred and ninety five cases of femoral head osteonecrosis in China. Int Orthop 40: 267-276, 2016.

2. Wang C, Peng J and Lu S: Summary of the various treatments for osteonecrosis of the femoral head by mechanism: A review. Exp Ther Med 8: 700-706, 2014.

3. Houdek MT, Wyles CC, Packard BD, Terzic A, Behfar A and Sierra RJ: Decreased osteogenic activity of mesenchymal stem cells in patients with corticosteroid-induced osteonecrosis of the femoral head. J Arthroplasty 31: 893-898, 2016.

4. Yeh CH, Chang JK, Ho ML, Chen CH and Wang GJ: Different differentiation of stroma cells from patients with osteonecrosis: A pilot study. Clin Orthop Relat Res 467: 2159-2167, 2009.

5. Balla B, Pintér C, Kósa JP, Podani J, Takács I, Nagy Z, Speer G, Horváth B, Korányi L and Lakatos P: Gene expression changes in femoral head necrosis of human bone tissue. Dis Markers 31: 25-32, 2011.

6. Wu X, Zhang Y, Guo X, Xu H, Xu Z, Duan D and Wang K: Identification of differentially expressed microRNAs involved in non-traumatic osteonecrosis through microRNA expression profiling. Gene 565: 22-29, 2015.

7. Zhao R, Li Y, Lin Z, Wan J, Xu C, Zeng Y and Zhu Y: miR-199b-5p modulates BMSC osteogenesis via suppressing GSK-3 $\beta / \beta$-catenin signaling pathway. Biochem Biophys Res Commun 477: 749-754, 2016.

8. Li T, Li H, Wang Y, Li T, Fan J, Xiao K, Zhao RC and Weng X: microRNA-23a inhibits osteogenic differentiation of human bone marrow-derived mesenchymal stem cells by targeting LRP5. Int J Biochem Cell Biol 72: 55-62, 2016.

9. Salmena L, Poliseno L, Tay Y, Kats L and Pandolfi PP: A ceRNA hypothesis: The Rosetta Stone of a hidden RNA language? Cell 146: 353-358, 2011.

10. Wang Q, Li Y, Zhang Y, Ma L, Lin L, Meng J, Jiang L, Wang L, Zhou $\mathrm{P}$ and Zhang Y: LncRNA MEG3 inhibited osteogenic differentiation of bone marrow mesenchymal stem cells from postmenopausal osteoporosis by targeting miR-133a-3p. Biomed Pharmacother 89: 1178-1186, 2017.

11. Weng J, Peng W, Zhu S and Chen S: Long noncoding RNA sponges miR-454 to promote osteogenic differentiation in maxillary sinus membrane stem cells. Implant Dent 26: 178-186, 2017.

12. Jeck WR, Sorrentino JA, Wang K, Slevin MK, Burd CE, Liu J, Marzluff WF and Sharpless NE: Circular RNAs are abundant, conserved, and associated with ALU repeats. RNA 19: 141-157, 2013.

13. Liu Q, Zhang X, Hu X, Dai L, Fu X, Zhang J and Ao Y: Circular RNA related to the chondrocyte ECM regulates MMP13 expression by functioning as a miR-136 'Sponge' in human cartilage degradation. Sci Rep 6: 22572, 2016. 
14. Dou C, Cao Z, Yang B, Ding N, Hou T, Luo F, Kang F, Li J, Yang X, Jiang H, et al: Changing expression profiles of lncRNAs, mRNAs, circRNAs and miRNAs during osteoclastogenesis. Sci Rep 6: 21499, 2016.

15. Ouyang Q, Wu J, Jiang Z, Zhao J, Wang R, Lou A, Zhu D, Shi GP and Yang M: Microarray expression profile of circular RNAs in peripheral blood mononuclear cells from rheumatoid arthritis patients. Cell Physiol Biochem 42: 651-659, 2017.

16. Peng W, Zhu S, Chen J, Wang J, Rong Q and Chen S: Hsa circRNA_33287 promotes the osteogenic differentiation of maxillary sinus membrane stem cells via miR-214-3p/Runx3. Biomed Pharmacother 109: 1709-1717, 2019.

17. Qian DY, Yan GB, Bai B, Chen Y, Zhang SJ, Yao YC and Xia H: Differential circRNA expression profiles during the BMP2-induced osteogenic differentiation of MC3T3-E1 cells Biomed Pharmacother 90: 492-499, 2017.

18. Otsuru S, Hofmann TJ, Olson TS, Dominici M and Horwitz EM: Improved isolation and expansion of bone marrow mesenchymal stromal cells using a novel marrow filter device. Cytotherapy 15 : 146-153, 2013.

19. Gao Y, Wang J and Zhao F: CIRI: An efficient and unbiased algorithm for de novo circular RNA identification. Genome Biol 16: 4, 2015.

20. Liu X, Shi X, Chen $C$ and Zhang L: Improving RNA-Sec expression estimation by modeling isoform- and exon-specific read sequencing rate. BMC Bioinformatics 16: 332, 2015.

21. Friedländer MR, Mackowiak SD, Li N, Chen W and Rajewsky N: miRDeep2 accurately identifies known and hundreds of novel microRNA genes in seven animal clades. Nucleic Acids Res 40: 37-52, 2012.

22. Livak KJ and Schmittgen TD: Analysis of relative gene expression data using real-time quantitative PCR and the 2(-Delta Delta C(T)) method. Methods 25: 402-408, 2001.

23. Gu W, Sun Y, Zheng X, Ma J, Hu XY, Gao T and Hu MJ Identification of gastric cancer-related circular RNA through microarray analysis and bioinformatics analysis. Biomed Res Int 2018: 2381680, 2018.

24. Song H, Tao L, Wang F, Wang W, Wei Y, Shen W and Zhou F: Effect of bone mesenchymal stem cells transplantation on the micro-environment of early osteonecrosis of the femoral head. Int J Clin Exp Pathol 8: 14528-14534, 2015.

25. Hernigou P, Beaujean F and Lambotte JC: Decrease in the mesenchymal stem-cell pool in the proximal femur in corticosteroid-induced osteonecrosis. J Bone Joint Surg Br 81: 349-355, 1999.

26. Song K, Huang M, Shi Q, Du T and Cao Y: Cultivation and identification of rat bone marrow-derived mesenchymal stem cells. Mol Med Rep 10: 755-760, 2014.

27. Yue J, Wan F, Zhang Q, Wen P, Cheng L, Li P and Guo W: Effect of glucocorticoids on miRNA expression spectrum of rat femoral head microcirculation endothelial cells. Gene 651: 126-133, 2018.

28. Han B, Chao J and Yao H: Circular RNA and its mechanisms in disease: From the bench to the clinic. Pharmacol Ther 187: 31-44, 2018.

29. Qu S, Yang X, Li X, Wang J, Gao Y, Shang R, Sun W, Dou K and Li H: Circular RNA: A new star of noncoding RNAs. Cancer Lett 365: 141-148, 2015.

30. Gu X, Li M, Jin Y, Liu D and Wei F: Identification and integrated analysis of differentially expressed lncRNAs and circRNAs reveal the potential ceRNA networks during PDLSC osteogenic differentiation. BMC Genet 18: 100, 2017.

31. Liao Q, Liu C, Yuan X, Kang S, Miao R, Xiao H, Zhao G, Luo H, $\mathrm{Bu} \mathrm{D}$, Zhao $\mathrm{H}$, et al: Large-scale prediction of long non-coding RNA functions in a coding-non-coding gene co-expression network. Nucleic Acids Res 39: 3864-3878, 2011

32. De Braekeleer E, Auffret R, Douet-Guilbert N, Basinko A, Le Bris MJ, Morel F and De Braekeleer M: Recurrent translocation $(10 ; 17)(\mathrm{p} 15 ; \mathrm{q} 21)$ in acute poorly differentiated myeloid leukemia likely results in ZMYND11-MBTD1 fusion. Leuk Lymphoma 55: 1189-1190, 2014.
33. Qi F, Hu JF, Liu BH, Wu CQ, Yu HY, Yao DK and Zhu L miR-9a-5p regulates proliferation and migration of hepatic stellate cells under pressure through inhibition of Sirt1. World J Gastroenterol 21: 9900-9915, 2015.

34. Lin CH, Li NT, Cheng HS and Yen ML: Oxidative stress induces imbalance of adipogenic/osteoblastic lineage commitment in mesenchymal stem cells through decreasing SIRT1 functions. J Cell Mol Med 22: 786-796, 2018.

35. Yuan HF, Zhai C, Yan XL, Zhao DD, Wang JX, Zeng Q, Chen L, Nan X, He LJ, Li ST, et al: SIRT1 is required for long-term growth of human mesenchymal stem cells. J Mol Med (Berl) 90: 389-400, 2012

36. Chen JS, Li HS, Huang JQ, Dong SH, Huang ZJ, Yi W, Zhan GF, Feng JT, Sun JC and Huang XH: MicroRNA-379-5p inhibits tumor invasion and metastasis by targeting FAK/AKT signaling in hepatocellular carcinoma. Cancer Lett 375: 73-83, 2016.

37. Sun C, Yuan H, Wang L, Wei X, Williams L, Krebsbach PH, Guan JL and Liu F: FAK promotes osteoblast progenitor cell proliferation and differentiation by enhancing Wnt signaling. J Bone Miner Res 31: 2227-2238, 2016.

38. Xu Y, Zhang G, Zou C, Zhang H, Gong Z, Wang W, Ma G, Jiang P and Zhang W: LncRNA MT1JP suppresses gastric cancer cell proliferation and migration through MT1JP/miR-214-3p/RUNX3 axis. Cell Physiol Biochem 46: 2445-2459, 2018

39. Wang Y, Feng Q, Ji C, Liu X, Li L and Luo J: RUNX3 plays an important role in mediating the BMP9-induced osteogenic differentiation of mesenchymal stem cells. Int J Mol Med 40: 1991-1999, 2017.

40. Huang C, Geng J, Wei X, Zhang R and Jiang S: miR-144-3p regulates osteogenic differentiation and proliferation of murine mesenchymal stem cells by specifically targeting Smad4. FEBS Lett 590: 795-807, 2016.

41. Gao W, Wu Y,He X, Zhang C, Zhu M, Chen B, Liu Q, Qu X, Li W, Wen S and Wang B: MicroRNA-204-5p inhibits invasion and metastasis of laryngeal squamous cell carcinoma by suppressing forkhead box C1. J Cancer 8: 2356-2368, 2017.

42. Hopkins A, Mirzayans F and Berry F: Foxc1 expression in early osteogenic differentiation is regulated by BMP4-SMAD activity. J Cell Biochem 117: 1707-1717, 2016.

43. Gu Y, Shi Y, Yang Q, Gu WW, He YP, Zheng HJ, Zhang X, Wang JM and Wang J: miR-3074-5p promotes the apoptosis but inhibits the invasiveness of human extravillous trophoblast-derived HTR8/SVneo cells in vitro. Reprod Sci 25: 690-699, 2018

44. Li Z, Jiang C, Li X, Wu WKK, Chen X, Zhu S, Ye C, Chan MTV and Qian W: Circulating microRNA signature of steroid-induced osteonecrosis of the femoral head. Cell Prolif 51, 2018 Doi: $10.1111 /$ cpr.12418

45. Zhang MY, Wang JB, Zhu ZW, Li LJ, Liu RS, Yang XK, Leng RX, Li XM, Pan HF and Ye DQ: Differentially expressed circular RNAs in systemic lupus erythematosus and their clinical significance. Biomed Pharmacother 107: 1720-1727, 2018.

46. Li LJ, Zhu ZW, Zhao W, Tao SS, Li BZ, Xu SZ, Wang JB, Zhang MY, Wu J, Leng RX, et al: Circular RNA expression profile and potential function of hsa_circ_0045272 in systemic lupus erythematosus. Immunology 155: 137-149, 2018.

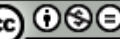

This work is licensed under a Creative Commons Attribution-NonCommercial-NoDerivatives 4.0 International (CC BY-NC-ND 4.0) License. 